\title{
On the role of gut bacteria and infant diet in the development of autoimmunity for type 1 diabetes
}

\author{
Arno L. M. Hänninen ${ }^{1}$ • Raine K. Toivonen ${ }^{1}$ \\ Received: 26 May 2015 / Accepted: 23 June 2015 /Published online: 11 July 2015 \\ (C) Springer-Verlag Berlin Heidelberg 2015
}

Keywords Bacteroidetes $\cdot$ Fermentable fibres $\cdot$ Gut immune system · Immune pathogenesis · Infant nutrition · Microbiota · Nonobese diabetic (NOD) mice · Type 1 diabetes

To the Editor: Environmental factors play a role in the development of type 1 diabetes. There is growing enthusiasm towards assessing the role of gut microbiota as an environmental modifier of autoimmunity in type 1 diabetes. This is largely based on the increasing awareness of the role of microbiota in regulating gut homeostasis and immune responses in the gut [1]. Microbiota composition varies between individuals and is modified by diet [2].

An emerging number of studies suggest that the abundance of Bacteroidetes is associated with autoimmunity in type 1 diabetes [3]. Thus, a diet promoting an abundance of Bacteroidetes could be an environmental risk modifier in type 1 diabetes. The notion of Bacteroides as 'diabetogenic' species is now further supported by a recent study in which Bacteroides dorei was found to be abundant in children who subsequently developed diabetes-related autoantibodies in a cohort of 76 children participating in the Diabetes Intervention and Prevention Project (DIPP) study in Southwest Finland [4].

Arno L. M. Hänninen arno.hanninen@utu.fi

1 Department of Medical Microbiology and Immunology, University of Turku, Kiinamyllynkatu 13, 20520 Turku, Finland
In NOD mice, diabetes incidence is effectively modified by diet [5]. We have studied the effect of two common nutritional fibres, pectin and xylan, on microbial colonisation, gut homeostasis and diabetes development in NOD mice [6]. Pectin and xylan dramatically increased colonisation of the intestine by Bacteroidetes, including B. dorei (Fig. 1 and data in [6]), increased the expression of stress-related molecular transcripts and inflammatory cytokines in the gut, and altered the balance of $\mathrm{T}$ cell cytokines in pancreatic lymph nodes. In terms of disease-promoting mechanisms, our findings alluded to a pathophysiological model [6] well aligned with the model recently discussed by Davis-Richardson and Triplett in Diabetologia [3]. The core idea is that, by displacing beneficial butyrate-producing taxa, Bacteroidetes have adverse effects on epithelial cells $[3,6]$. Our findings provided experimental evidence in support of this and indicated that at least some Bacteroides species play a role in diabetes-related autoimmunity. In addition, our results identified dietary fibres as novel candidates for environmental modifiers of the pathogenesis of diabetes. Interestingly, induction of low-grade inflammation in the gut and microbiota encroachment was recently reported in mice following administration of two other fibre compounds used as dietary emulsifiers [7].

According to Finnish healthcare recommendations, fermentable fibres including pectin and xylan (hemicellulose, a constituent of all plant cell walls) are introduced into the diet of infants in the form of berry cocktails. In Finland, parents are recommended to start giving berry cocktails to their infants at 4 months age, an age preceding the peak in the observed abundance of B. dorei [3]. 

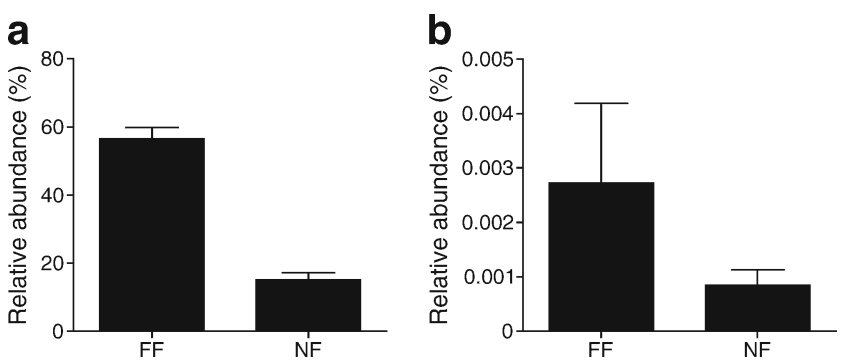

Fig. 1 Dietary fermentable fibres increase the amount of Bacteroidetes species in the gut. (a) The relative abundance of members of the Bacteroidetes phylum is increased in mice fed a diet containing fermentable fibres compared with control mice fed a diet containing non-fermentable fibres. (b) The same trend is also observed for the prevalence of B. dorei. Mice were fed with semisynthetic diets containing either pectin and xylan (fermentable fibres) and cellulose, or cellulose (non-fermentable or 'insoluble' fibre) alone (total fibre content was equal in both groups) for 4 weeks from the time of weaning. Bacterial DNA was extracted from colon content and sequenced using high-throughput sequencing methods (Illumina Miseq) targeted for 16S rRNA gene variable regions V4-V5 $(n=4, * * * * p<0.0001)$

Bacteroidetes are capable of adapting to different nutritional environments and they possess a large number of genes that encode carbohydrate-active enzymes. After weaning, in the murine intestine, Bacteroidetes start to express a plethora of enzymes that participate in the liberation of simple sugars from complex polysaccharides of dietary fibres [8]. Simultaneously, the expression of genetic loci encoding enzymes involved in the synthesis of capsular polysaccharides is modified [8].

The immune-stimulating and metabolic effects of different members of the microbiota are still incompletely understood, and differences are likely between different individuals' bacteria representing even the same species, including $B$. dorei [3]. We envision that fermentable fibres may modify the function and immunogenicity of microbiota, including members of the Bacteroidetes phylum, in ways which may have relevance to type 1 diabetes [6].
Funding Work by the authors is funded by Päivikki and Sakari Sohlberg Foundation, Diabetes Research Foundation Finland and the Academy of Finland.

Duality of interest The authors declare that there is no duality of interest associated with this manuscript.

Contribution statement All authors were responsible for drafting the article and revising it critically for important intellectual content. All authors approved the version to be published.

\section{References}

1. Hooper LV, Littman DR, Macpherson AJ (2012) Interactions between the microbiota and the immune system. Science 336:12681273

2. Scott KP, Gratz SW, Sheridan PO, Flint HJ, Duncan SH (2013) The influence of diet on the gut microbiota. Pharmacol Res 69: $52-60$

3. Davis-Richardson AG, Triplett EW (2015) A model for the role of gut bacteria in the development of autoimmunity for type 1 diabetes. Diabetologia 58:1386-1393

4. Davis-Richardson AG, Ardissone AN, Dias R et al (2014) Bacteroides dorei dominates gut microbiome prior to autoimmunity in Finnish children at high risk for type 1 diabetes. Front Microbiol 5: 678

5. Coleman DL, Kuzava JE, Leiter EH (1990) Effect of diet on incidence of diabetes in nonobese diabetic mice. Diabetes 39: $432-436$

6. Toivonen RK, Emani R, Munukka E et al (2014) Fermentable fibres condition colon microbiota and promote diabetogenesis in NOD mice. Diabetologia 57:2183-2192

7. Chassaing B, Koren O, Goodrich JK et al (2015) Dietary emulsifiers impact the mouse gut microbiota promoting colitis and metabolic syndrome. Nature 519:92-96

8. Bjursell MK, Martens EC, Gordon JI (2006) Functional genomic and metabolic studies of the adaptations of a prominent adult human gut symbiont, Bacteroides thetaiotaomicron, to the suckling period. J Biol Chem 281:36269-36279 